\title{
Paroxysmal atrial fibrillation recognition based on multi-scale Rényi entropy of ECG
}

\author{
Yi Xin*, Yizhang Zhao, Yuanhui Mu, Qin Li and Caicheng Shi \\ School of Life Sciences, Beijing Institute of Technology, Beijing, China
}

\begin{abstract}
.
BACKGROUND: Atrial fibrillation (AF) is a common type of arrhythmia disease, which has a high morbidity and can lead to some serious complications. The ability to detect and in turn prevent AF is extremely significant to the patient and clinician. OBJECTIVE: Using ECG to detect AF and develop a robust and effective algorithm is the primary objective of this study. METHODS: Some studies show that after AF occurs, the regulatory mechanism of vagus nerve and sympathetic nerve will change. Each R-R interval will be absolutely unequal. After studying the physiological mechanism of AF, we will calculate the Rényi entropy of the wavelet coefficients of heart rate variability (HRV) in order to measure the complexity of PAF signals, as well as extract the multi-scale features of paroxysmal atrial fibrillation (PAF).

RESULTS: The data used in this study is obtained from MIT-BIH PAF Prediction Challenge Database and the correct rate in classifying PAF patients from normal persons is $92.48 \%$.

CONCLUSIONS: The results of this experiment proved that AF could be detected by using this method and, in turn, provide opinions for clinical diagnosis.
\end{abstract}

Keywords: Atrial fibrillation, heart rate variability, wavelet, entropy

\section{Introduction}

Atrial fibrillation (AF) is a common type of arrhythmia diseases. It has high morbidity rates with the ability to induce strokes, thrombus, and many other serious complications [1]. Nearly half of the individuals that suffer from AF suffer from paroxysmal atrial fibrillation (PAF) and after repeated occurrence of AF, these patients always turn to persistent atrial fibrillation. Studies show that the change in vagus nerve may be an important factor related to the onset and termination of PAF. Therefore, the analysis of heart rate variability (HRV) signals [2] has been regarded as a non-invasive, but effective method of reflecting the status of autonomic nervous systems.

The main methods used in HRV analysis are time-domain, frequency domain, and non-linear [3-7]. The time-domain method proposes limitations on the fluctuation of heart rate. The frequency-domain method is solely based on a steady model; therefore it cannot show important detailed characteristics of HRV. In recent years, wavelet transform and empirical mode decomposition (EMD) have been widely used. They analyze signal's time-frequency characteristics $[6,7,18]$. Wavelet transform can decompose the signal into different frequency bands by convolving a signal with a wavelet basis function, leading to

\footnotetext{
${ }^{*}$ Corresponding author: Yi Xin, School of Life Sciences, Beijing Institute of Technology, 5 south Zhongguancun street, Haidian District 100081, Beijing, China. Tel.: +86 13810028162; E-mail: ameko@bit.edu.cn.
}

0928-7329/17/ $\$ 35.00$ C 2017 - IOS Press and the authors. All rights reserved

This article is published online with Open Access and distributed under the terms of the Creative Commons Attribution NonCommercial License (CC-BY-NC 4.0). 

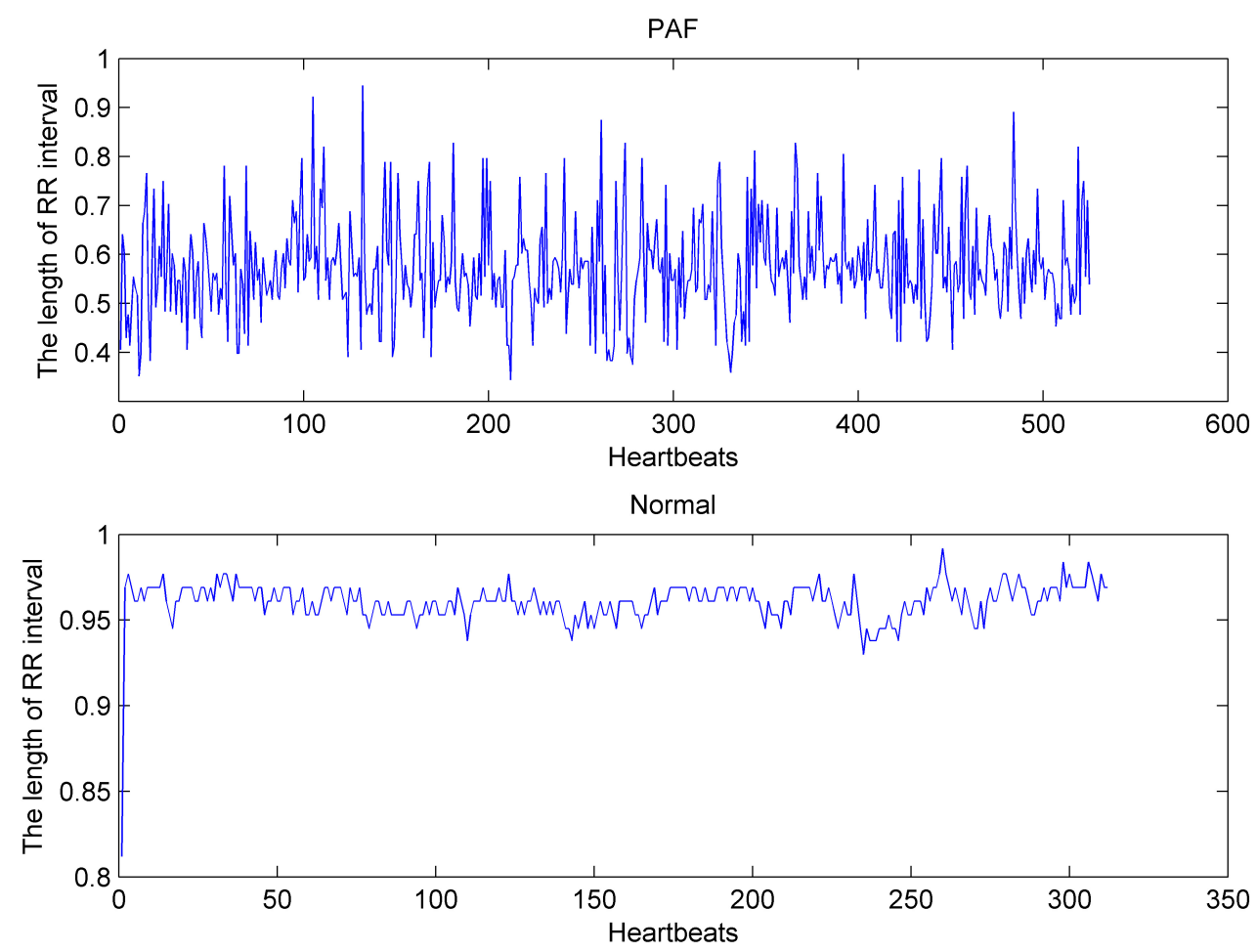

Fig. 1. HRV signals of PAF patients and normal people.

good time-frequency localization ability. The $0.04 \sim 0.15 \mathrm{~Hz}$ low frequency part of HRV reflects the activity intensity of sympathetic nerve, while the $0.15 \sim 0.4 \mathrm{~Hz}$ high frequency part shows the erethism of vagus nerve [7]. Because of these reasons, the multiscale analyses based on wavelet transform were used extensively in ECG signal processing.

The HRV signal of healthy people can show slight fluctuations, but HRV signals are nonlinear and not stationary. The two figures shown are the p08c signal and the n08c signal from the MIT-BIH PAF Prediction Challenge Database. As shown in Fig. 1, the fluctuations will become violent and chaotic when PAF occurs. Nowadays, the chaotic theory is being widely used. Rudolf Clausius developed entropy, which has been used to signal the analysis area to characterize the complexity of a system or signal [16]. The analysis of fractal structure and complexity of HRV signals based on entropy [5,9,12] and other nonlinear methods have been extremely popular throughout the world. Wavelet transform methods lacked the analysis of the chaos of HRV signals. The nonlinear analysis lacked detailed information because it could only show chaos on the whole level; therefore, we considered proposing a method combining both of them.

\section{Method}

Wavelet transform has been widely used in the analysis of HRV signals since Vetterli first applied it in filter [17]. Therefore, we have the ability to obtain different frequency signals by using wavelet transform. Entropy can describe the chaos levels of a signal as well as measure the level of complexity in the nonlinear dynamics area $[9,12]$. Zero entropy denotes that the system is well regulated, while 
infinity entropy means that the system is completely chaotic. The Rényi entropy [10] is a generalized form of Shannon and other kinds of entropy, but the Rényi entropy often has a better performance than any of them. Based on the physiological characteristics of PAF signal, in order to extract features of HRV signal we proposed a multi-scale Rényi entropy by combining both the wavelet transform and Rényi entropy methods. After this, in order to detect and determine PAF signals with health signals, as well as signals that are far away from PAF, we used the support vector machine (SVM) method.

\subsection{Multi-scale Rényi entropy}

Because the wavelet analysis relies on multi-scale analysis, it reflect the potential information of HRV signals much better when combined with Rényi entropy calculation. The procedures of the proposed method are listed below:

- First, make m-scale discrete wavelet transform on HRV signals. The discrete wavelet function $\varphi_{j, k}(t)$ is often described as:

$$
\varphi_{j, k}(t)=2^{-\frac{j}{2}} \varphi\left(2^{-j} t-k\right)
$$

Where $j$ denotes the scale factor, and $k$ is shift factor.

Thus, the discrete wavelet coefficients $C_{j k}$ of HRV signals were obtained:

$$
C_{j k}=\int_{-\infty}^{\infty} f(t) \varphi_{j, k}^{*}(t) d t=\left\langle f, \varphi_{j, k}\right\rangle
$$

Where $\varphi_{j, k}^{*}(t)$ is the conjugate number of $\varphi_{j, k}(t)$.

If the wavelet coefficient vector on scale $j$ is called $W_{j}$ :

$$
W_{j}=\left(C_{j 1}, C_{j 2}, \ldots, C_{j n}\right)
$$

Then $n$ would denote the length of the wavelet coefficient on this scale.

- Mixing the wavelet coefficient on scale $\mathrm{j}$, the Rényi entropy of order $q$, where $q \geqslant 0$ and $q \neq 1$, is defined as:

$$
H\left(W_{j}\right)=\frac{1}{1-q} \ln \sum_{k=1}^{n}\left(p_{j k}\right)^{q}
$$

$p_{j k}$ is described as:

$$
p_{j k}=C_{j k} / \sum_{k=1}^{n} C_{j k}
$$

- Here the Rényi entropy is a generalized form of Shannon and other kinds of entropy. Rényi entropy with different parameter $q$ has a different performance. In order to find the best results, we should choose a method to find the most appropriate $q$. In this article, the traversing method was applied.

\subsection{Implement method}

In this paper, we proposed a multi-scale Rényi entropy method for feature extraction of HRV signals and then applied it on PAF recognition and classification. In order to obtain HRV signals, we first have to complete preprocessing on the original ECG signals to remove the noises. Then to accurately specify PAF, we extract the different characteristics of HRV signals using Rényi entropy. Last, a classifier will be trained with the train dataset to classify the test dataset by the feature of signals. The proposed method is described in Fig. 2: 


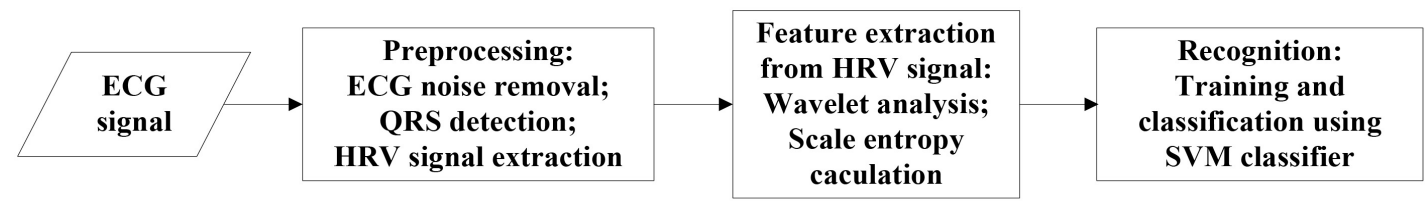

Fig. 2. Algorithm flow chart.

1. Data Preprocessing: Power line interference, electrical interference, and baseline wander noises often interfered with the original ECG signal noises. First of all, a $5 \sim 15 \mathrm{~Hz}$ band pass filter is designed to remove noises, then the Pan-Tompkins [14] method is applied for the positioning of $\mathrm{R}$ waves. By calculating the length of the intervals of adjacent $\mathrm{R}$ waves, the original HRV signals will be determined.

2. Feature extraction: First, select the best wavelet function to decompose signal based on the characteristics of HRV. Then choose order q and calculate the Rényi entropy of each scale as figure 1.1 mentioned. By doing this, we were able to obtain the feature vectors for classification.

3. Recognition of PAF: Use SVM to classify selected features of PAF signals into two groups with normal and far PAF signals. The kernel function is a Radial Basis Function.

\section{Results and discussion}

In this paper, to verify our method we used the MIT-BIH PAF Prediction Challenge Database. The entire dataset includes 50 normal ECG signals, 25 normal PAF signals, and 25 signals that are far away from PAF. In order to classify as a signal far away from PAF, the sample must not have any PAF occurring within 45 minutes before and after sampling signals. A normal signal is marked with the letter $\mathrm{n}(\mathrm{n} 08)$. A signal coming from a patient begins with the letter $\mathrm{p}$ and ends with the letter $\mathrm{c}$. An odd middle digit indicates that the signal is far away from PAF. On the contrary, the signal is a PAF signal if it is even. All signals are five minutes long and the sampling frequency is $128 \mathrm{~Hz}$.

First, as described above, a $5 \sim 15 \mathrm{~Hz}$ band pass filter was designed to remove the noises. Then the Pan-Tompkins method was applied for positioning of the $\mathrm{R}$ waves so that the five-minute length HRV signals could be obtained. Because of the advantages of Daubechies wavelet, the db8 wavelet was applied as the wavelet base function. This resulted in an eight-layer decomposition on the HRV signals to analyze the excitability of cardiac electrical activity on different scale coefficients. Then the Rényi entropy of each scale coefficients was calculated and the SVM method was used for classification.

Three statistics were calculated to show the results of the classification: correct rate, sensitivity, and specificity [15].

$$
\begin{aligned}
& \text { Correct rate }(\%)=\frac{\mathrm{TP}+\mathrm{TN}}{\mathrm{TP}+\mathrm{TN}+\mathrm{FN}+\mathrm{FP}} \times 100 \\
& \text { Sensitivity }(\%)=\frac{\mathrm{TP}}{\mathrm{TP}+\mathrm{FN}} \times 100 \\
& \text { Specificity }(\%)=\frac{\mathrm{TN}}{\mathrm{TN}+\mathrm{FP}} \times 100
\end{aligned}
$$

The five-fold-cross-validation method was employed 100 times per classification in order to avoid excessive fitting and improve upon the credibility of the classification results. As mentioned previously, in order to get a better classification results, the most appropriate $q$ needed to be clarified; a number 
Table 1

Comparing the features (the entropy of each scale) of Normal signal and PAF signal

\begin{tabular}{cccc}
\hline Level & Normal & PAF & T-test $(\mathrm{p}$-value $)$ \\
\hline L1 & -15.9187 & -19.0163 & $7.3338 \mathrm{e}-06$ \\
L2 & -15.0825 & -18.1880 & $2.6657 \mathrm{e}-06$ \\
L3 & -14.7346 & -17.3493 & $3.0438 \mathrm{e}-05$ \\
L4 & -14.5609 & -17.0870 & $5.5874 \mathrm{e}-05$ \\
L5 & -14.6049 & -16.2469 & 0.0047 \\
L6 & -14.6287 & -16.4229 & 0.0036 \\
L7 & -14.5701 & -16.5680 & 0.0026 \\
L8 & -14.6940 & -16.6299 & 0.0051 \\
\hline
\end{tabular}
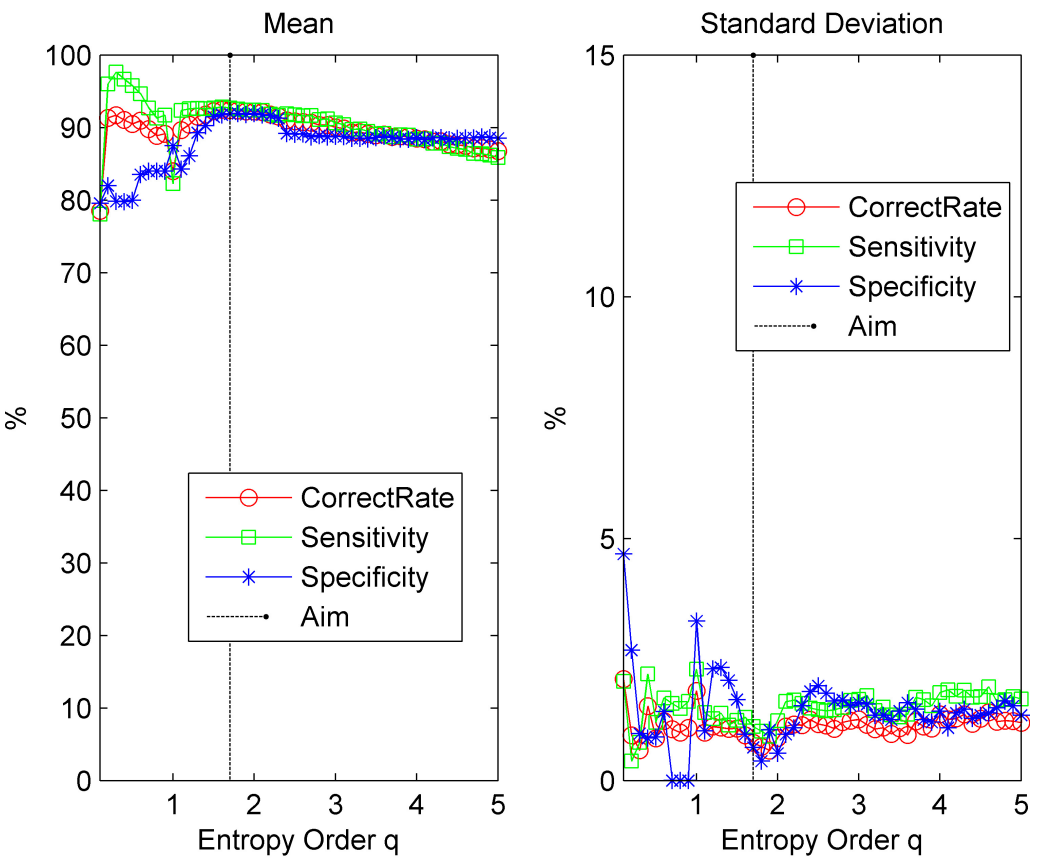

Fig. 3. Classification results of PAF and normal signals.

between $0 \sim 5$ was generally considered. In this paper, directly traversing method that cross from 0.1 to 5.0 was designed, and the step length was set to 0.1 .

Figure 3 shows the results of the first experiment where a classification of 50 normal HRV signals and 25 PAF signals was conducted. The left chart indicates the average values of the correct rate, sensitivity, and specificity of the classification. The right chart indicates the standard deviation of these values. Figure 3 suggests that the results were quite steady when $q=1.7$; the correct rate reaches the highest, and the standard deviation was the lowest. The correct rate, sensitivity, and specificity were $(92.48 \pm$ $0.13) \%,(92.76 \pm 0.21) \%$, and $(91.76 \pm 0.12) \%$, respectively.

With the parameter set at $q=1.7$, two-sided student's t test has been used in the experimental result in order to ensure the reliability of the results. The confidence level of Student's t test is 0.05. L1-L8 represents eight scale of wavelet decomposition respectively in Table 1.

In Table 1, the $\mathrm{P}$ value reflects the otherness of the entropy in the same scale between two samples. $P<$ 0.05 means that the two samples have statistical differences and $0.01<p<0.05$ means the statistical 
Table 2

Comparing the features (the entropy of each scale) of Far away from PAF signals and PAF signals

\begin{tabular}{cccc}
\hline Level & Far away from PAF & PAF & T-test (p-value) \\
\hline L1 & 17.3134 & 19.7883 & $2.8482 \mathrm{e}-08$ \\
L2 & 15.9941 & 18.3143 & $2.6428 \mathrm{e}-08$ \\
L3 & 14.4490 & 16.8167 & $2.9309 \mathrm{e}-08$ \\
L4 & 13.6736 & 15.8650 & $1.5506 \mathrm{e}-07$ \\
L5 & 12.9384 & 14.8009 & $8.1806 \mathrm{e}-07$ \\
L6 & 12.3516 & 14.3659 & $8.7663 \mathrm{e}-07$ \\
L7 & 11.6949 & 14.0699 & $4.7931 \mathrm{e}-09$ \\
L8 & 11.4185 & 13.9405 & $1.1491 \mathrm{e}-08$ \\
\hline
\end{tabular}
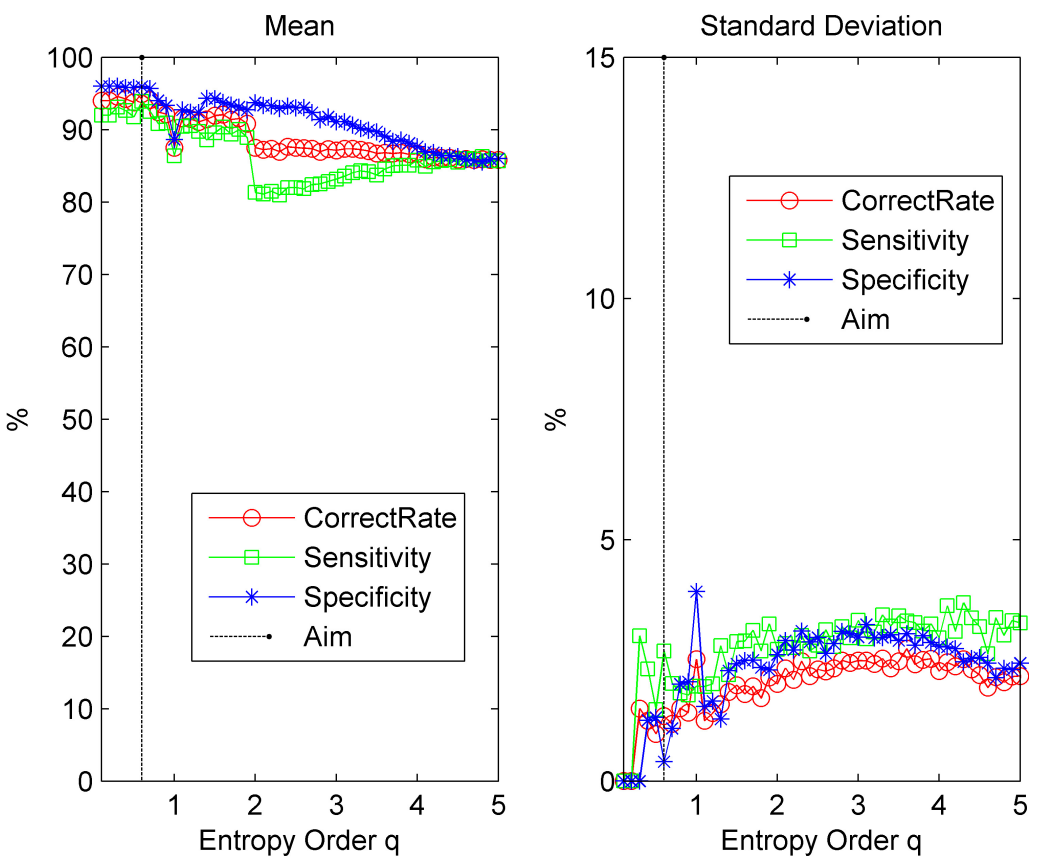

Fig. 4. Classification results of PAF and signals far away from PAF.

differences are very evident. In order to use the feature to classify the normal and PAF signals, the Multi-scale Rényi entropy value has significant different. The physiological reason that the excitability and complexity of vagus rises when PAF occurred can be proven is because the absolute value of entropy of PAF signal is higher than the value of normal signal in every scale. Therefore, the entropy will rise with the increase of complexity.

Then, the same experiment of 25 PAF signals and 25 signals far away from PAF was completed. The left chart in Fig. 4 indicates the mean values of the correct rate, sensitivity, and specificity of the classification and the right chart indicates is the standard deviation of these values. In Fig. 4 we can find that when $q=0.6$ the classification results were the best. As q grows, the results worsen, and the standard deviation increases. The correct rate, sensitivity, and specificity were $(94.90 \pm 0.12) \%,(93.84 \pm 0.33) \%$ and $(95.96 \pm 0.19) \%$, respectively.

In this experiment, the two-sided student's t test was also used to ensure that the results were reliable. In Table 2, the value of parameter was set to 0.6. L1-L8 represents eight scale of wavelet decomposition. 
The $\mathrm{P}$ value in Table 2 shows the otherness of the entropy in the same scale between the two samples. It shows that in every scale, the entropy value of PAF signal is much higher than the far away from PAF value. It also related with the complexity of vagus activity.

\section{Conclusions}

In this paper, we obtained satisfying results by combined the wavelet analysis with nonlinear entropy in order to propose a feature extraction algorithm for PAF recognition. The disadvantages of traditional time-frequency analysis can be overcome by calculating the Rényi entropy of different scales. Considering the physiological characteristics of PAF, the Multi-scale Rényi entropy combines the multi resolution and local feature ability of Wavelet Transform with the entropy in information theory. By doing this, the characteristics of vagus nerve activity will be quantified. With our experimental approach, we were able to successfully classify the PAF signals with normal or far away signals. The results presented throughout this paper show that the application of this method is successful in real-time monitoring of $\mathrm{AF}$ patients, as well as prediction and diagnosis of PAF.

\section{Acknowledgments}

This work is supported by the Funding for Basic Research in Beijing Institute of Technology.

\section{Conflict of interest}

None to report.

\section{References}

[1] Wolf PA, Abbott RD, Kannel WB. Atrial fibrillation as an independent risk factor for stroke: the Framingham Study. Stroke; a journal of cerebral circulation. 1991; 22(8): 983-8.

[2] Omerbegovic M. Analysis of Heart Rate Variability and Clinical Implications. Medical Archives. 2009; 63(2): 102-5.

[3] Evrengül H, Tanriverdi H, Dursunoglu D, et al. Time and frequency domain analyses of heart rate variability in patients with epilepsy. Epilepsy Research. 2005; 63(2-3): 131-139.

[4] Bilgin S, Çolak OH, Koklukaya E, et al. Efficient solution for frequency band decomposition problem using wavelet packet in HRV. Digital Signal Processing. 2008; 18(6): 892-899.

[5] Shin DG, Yoo CS, Yi SH, et al. Prediction of paroxysmal atrial fibrillation using nonlinear analysis of the R-R interval dynamics before the spontaneous onset of atrial fibrillation. Circulation Journal. 2006; 70(70): 94-99.

[6] Zhang AH, Kou MC, Diao C, et al. Quality Assessment of ECG Signal Based on Wavelet Energy Ratio and Wavelet Energy Entropy. Applied Mechanics \& Materials. 2014; 530-531: 577-580.

[7] İşler Y, Kuntalp M. Combining classical HRV indices with wavelet entropy measures improves to performance in diagnosing congestive heart failure. Computers in Biology \& Medicine. 2007; 37(10): 1502-1510.

[8] Asl BM, Setarehdan SK, Mohebbi M. Support vector machine-based arrhythmia classification using reduced features of heart rate variability signal. Artificial Intelligence in Medicine. 2008; 44(1): 51-64.

[9] Bian CH, Qianli MA, Junfeng SI, et al. Sign series entropy analysis of short-term heart rate variability. Chinese Science Bulletin. 2009; 54(24): 4610-4615.

[10] Kulish V, Sourin A, Sourina O. Human electroencephalograms seen as fractal time series: Mathematical analysis and visualization. Computers in Biology \& Medicine. 2006; 36(3): 291-302.

[11] Wu GQ, Arzeno NM, Shen LL, et al. Chaotic signatures of heart rate variability and its power spectrum in health, aging and heart failure. Plos One. 2009; 4(2): e4323-e4323. 
[12] Masè M, Faes L, Antolini R, et al. Quantification of synchronization during atrial fibrillation by Shannon entropy: validation in patients and computer model of atrial arrhythmias. Physiological Measurement. 2005; 26(6): 911-23.

[13] Balocchi R, Menicucci D, Santarcangelo E, et al. Deriving the respiratory sinus arrhythmia from the heartbeat time series using empirical mode decomposition. Chaos Solitons \& Fractals. 2004; 20(1): 171-177.

[14] Pan J, Tompkins WJ. A Real-Time QRS Detection Algorithm. IEEE Transactions on Biomedical Engineering. 1985; BME-32(3): 230-236.

[15] Yeh YC, Che WC, Lin HJ. Analyzing ECG for cardiac arrhythmia using cluster analysis. Expert Systems with Applications. 2012; 39(1): 1000-1010.

[16] Cropper WH. Rudolf Clausius and the road to entropy. American Journal of Physics. 1986; 54(12): 1068-1074.

[17] Vetterli M, Herley C. Wavelets and filter banks: theory and design. IEEE Transactions on Signal Processing. 1992; 40(9): 2207-2232.

[18] Xin Y, Chen Y, Hao W. ECG Baseline Wander Correction Based on Mean-median Filter and Empirical Mode Decomposition. Bio-medical Materials and Engineering. 2014; 24(1): 365-71. 